\title{
Scientific Evidence of the Progressive Increase in Posmastoplasty Augmentation Capsule Contracture with Implants
}

\section{Tamayo Carbon $\mathrm{AM}^{1}$, Vila Garcia E ${ }^{2 *}$, Manrique Trujillo $\mathrm{AF}^{3}$ and Cuastumal} Figueroa $\mathrm{DK}^{4}$

${ }^{1}$ Specialists of II degree in Plastic Surgery and Caumatology, Surgical Hospital Hermanos Ameijeiras, Cuba

${ }^{2}$ Specialist I degree in General Surgery, Surgical Hospital Hermanos Ameijeiras, Cuba

${ }^{3} 4$ th year Resident of Plastic Surgery and Caumatology, Surgical Hospital Hermanos Ameijeiras, Cuba

${ }^{4} 1$ st year Resident of Plastic Surgery and Caumatology, Surgical Hospital Hermanos Ameijeiras, Cuba

*Corresponding author: Eyleen Vila Garcia, Services of Plastic Surgery and Caumatology and General Surgery, Surgical Hospital Hermanos Ameijeiras, Cuba, Tel: 53232327; Email: hielenvg@infomed.sld.cu

\section{Abstract}

A health problem is exposed that has a high incidence and that in many cases can be preventable. The etiology and symptoms of capsular contracture as the most frequent complication of augmentation mastoplasty are explained. This research is carried out with the aim of identifying the risk factors associated with the formation of capsular contracture, as well as its main causes. Health education for patients who undergo augmentation mastoplasty guarantees a successful recovery. Plastic surgeons are required to apply national action protocols to avoid the appearance of this deforming complication, the treatment of which requires surgical reoperations with possible recurrences that have an unfavorable economic and social impact.

Keywords: Mastoplasty; Implants; Capsular Contracture

\section{Introduction}

Breast augmentation surgery with the use of implants is a common procedure in Plastic and Reconstructive Surgery and Capsular Contracture (CC) is the most frequent complication and cause of reoperation [1,2]. The formation of fibrous tissue around the implant is a physiological process, but its excessive production and contraction causes CC, which is expressed by distortion of the breast, hardening and pain. This complication is reported between 5 and 25\% of cases [3]. The adequate diagnosis of the condition is based on the Baker classification and stages III and IV are relevant because they require reoperation $[3,4]$. The causes and etiopathogenesis of the process are not they are well identified, although most of the current evidence suggests an immune-based mechanism. Historically, its presence is associated with a local inflammatory reaction due to the presence of postoperative hematomas and infections mainly related to staphylococcus [5]. Other hypotheses that refer to the origin of this process point to the periareolar incision when the implant is placed, the smooth surface of the same, its location in the subglandular plane and the longest evolution time of having placed it $[1,3]$.

Researchers on the subject also consider the existence of leaks of the prosthetic material as a possible factor associated with its appearance. It is proposed that the rupture of the prosthesis causes cystic fibrosis and CC [2]. Plastic surgeons have to develop strategies to avoid this complication based on the identified risk factors, to help 


\section{International Journal of Transplantation \& Plastic Surgery}

enhance the prevention actions of CC secondary to implant augmentation mastoplasty.

There is an increase in patients with this complication, so an alert is made about modifiable factors that can be intervened to prevent it. With the aim of preventing its appearance, the main causes of CC are disclosed and the health education of patients undergoing augmentation mastoplasty is emphasized for their proper recovery. Plastic surgeons must be strict in applying established surgical protocols. It is a task for all surgeons to combat medical intrusion to prevent CC with its fatal economic and social consequences.

\section{Results}

In Cuba in the last decade there has been an increase in the demand for augmentation mastoplasty and also for patients with CC. This is a social problem with a negative economic impact, since it requires readmissions, the majority of implants have to be changed and prolonged rest with a high risk of recurrence. Several cases with different characteristics are reported in relation to the type of implant, the incision made and its location, but cases with a subglandular location predominate.

Under normal conditions, the placement in our body of a foreign body, such as prosthesis, stimulates the immune system to try to isolate it by forming a capsule around it. This capsule is a thin, shiny white envelope that does not affect the location or shape of the breast implant. However, different intrinsic and extrinsic factors can overstimulate this immune reaction, giving rise to a thick capsule, with increased surface tension, capable of deforming the breast prosthesis and causing pain (Figure 1).

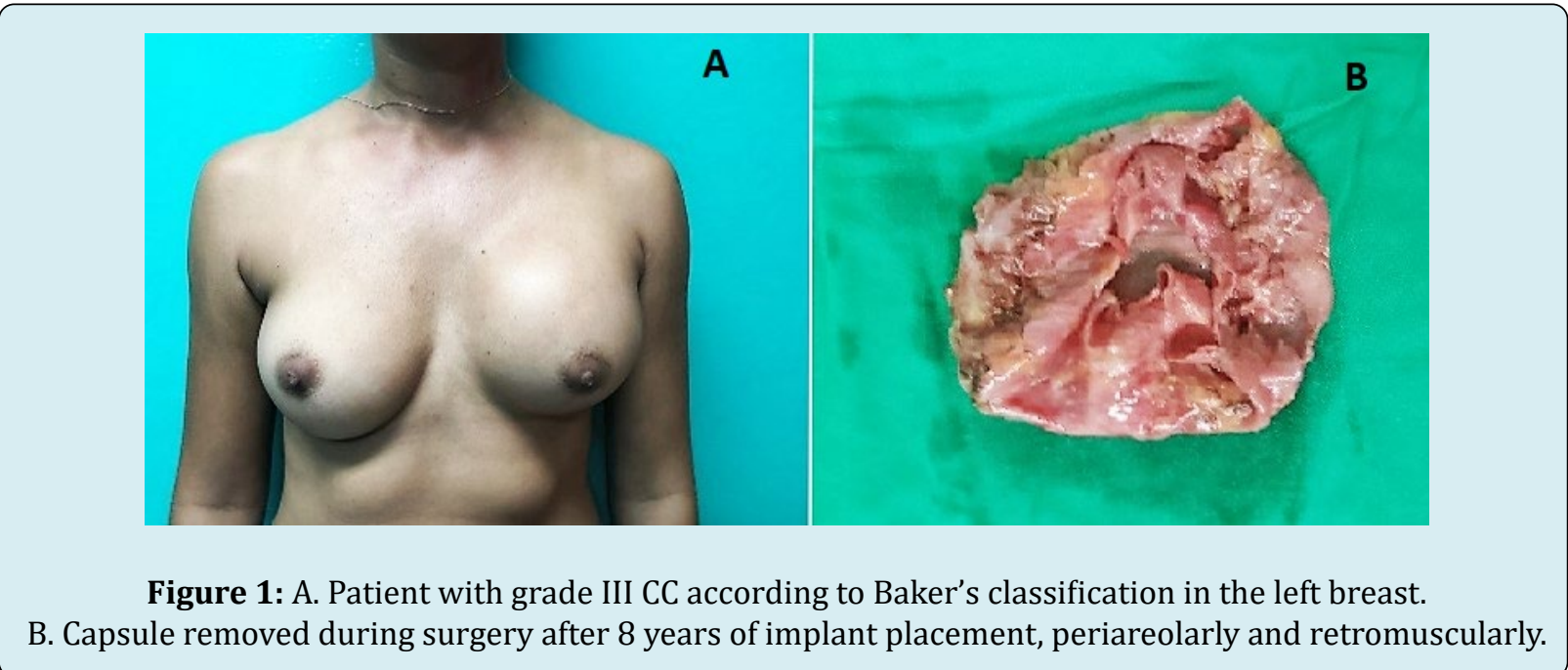

The Hermanos Ameijeiras Hospital is of tertiary care level, so it receives patients from all over the country with complex diseases that are difficult to treat. In the last 5 years, in the Plastic Surgery Service, around 200 augmentation Mastoplasty surgeries were performed annually, with an average incidence of CC of $5 \%$ of the patients. These results are encouraging and are due to the fact that the plastic surgeons dedicated to Mastology make the adequate selection of the patients, their health education as a fundamental pillar of the postoperative recovery, the selection of the incision according to the characteristics of each patient with predilection for the submammary sulcus, the use of textured implants and the placement plane of the submuscular implant. However, the high incidence of CC in other hospitals in the country is up to $15 \%$, with a tendency to progressive increase and due to their difficult treatment, they are referred to our institution.

\section{Discussion}

Current studies do not clearly define the etiology of the process. It is known that it is an entity of multifactorial origin, but the catalyst of the inflammatory process is not clear. Meta-analyzes indicates subglandular implant placement, periareolar incision, and smooth implant characteristics as repeated risk factors [1-3].

In investigations carried out, an association was found between the appearance of $\mathrm{CC}$ and the skilled hand that required upper limb effort, postoperative for more than 6 years, and augmentation mastoplasty with a periareolar approach. The prevention of this complication is emphasized, because its treatment is surgical and difficult to solve [6]. 


\section{International Journal of Transplantation \& Plastic Surgery}

Currently the incidence of CC is estimated between 10 and $15 \%$ without other risk factors. However, there are factors associated with the patient, the surgical technique, and the type of breast implant that can even double the risk of CC [7].

\section{Patient Factors}

The intrinsic factors of the patients that depend on their scar process and their immune response do not allow exerting a direct influence on them, but they can be avoided with an adequate selection of patients for surgery. The safety criteria for augmentation mastoplasty are having no history of immune or connective tissue disease, chemotherapy or radiation therapy, or the presence of late, acute, or subclinical infections. Bacteria that are present on the skin and in the breast ducts create a film around the implant that acts as a continuous irritant and causes a thicker capsule. The profession that requires effort of the upper limbs with physical exercise produces friction between the capsule and the rough implant, which generates small breaks in the periprosthetic capsule and the subsequent inflammatory process. Mastitis produced during breastfeeding in bovine studies has demonstrated the presence of Staphylococcus xyllosus that produce bacterial endotoxins, enzymes, and biofilms. In postoperative care, massage of the breast prevents contracture due to disruption of the postoperative capsular formation due to the anti-inflammatory effect of this displacement, the appearance of hematomas or seromas, microtrauma causes an exudates that increases in size until reaching the limit point for its reabsorption, known as late seroma, a situation that would be the reason why this space gradually increases in size, leading to the development of contracture [8].

\section{Surgical Factors}

The periareolar approach, since when it is introduced through this route, contact with the milk ducts that communicate with the outside increases the risk of infection. Given the poor vascularization of this plane, the placement of the prosthesis in the subglandular plane is known to greatly increase the encapsulation rate and induces the formation of firmer capsules. However, the submuscular placement favors, due to the massage performed by the pectoralis major muscle on the implant, that the capsule remains soft. The making of the pocket, a small space for a large implant, leads to the formation of folds on the surface of the prosthesis that favor the development of CC due to the disproportion between the content and the continent that generates tension [9] (Figure 2).

\begin{tabular}{|l|}
\hline Administration of intravenous antibiotics before and dur ing the intervention \\
\hline Cleaning the skin with antiseptics such as povidone iodine or chlorhexidine \\
\hline Cov er the areola nipple compl ex with a protective film to avoid local contamination \\
\hline Incisions in the submammary sulc us whenever possible bec ause they have a lower incidence \\
\hline Careful dissection with an electric sc alpel, to avoid bleeding \\
\hline Avoid dissection witlin the parenc lymm of the breast \\
\hline $\begin{array}{l}\text { Placement of the impl ant in the sulmus cular plane bec ause it has been shown to have a lower } \\
\text { incidence of contracture than the subglandular }\end{array}$ \\
\hline Inigate pocket with antibiotic solution \\
\hline Clange gloves and surgical instruments at the time of prosthetic insertion \\
\hline Use a sleeve to insert the implant \\
\hline Minimize the time of exposure of the breast implant \\
\hline Careful introduction of the implant, without deforming or compressing it excessively \\
\hline Minimize manipulation of the breast implant once placed \\
\hline Meticulous hemostasis \\
\hline \hline Wound closure by planes \\
\hline \hline Avoid using drains \\
\hline Posterior antibiotic prophylaxis protocol \\
\hline
\end{tabular}

Figure 2: Promotion and prevention of $\mathrm{CC}$ on factors that depend on surgical technique. 


\section{International Journal of Transplantation \& Plastic Surgery}

\section{Factors Associated with Breast Implant}

There is a greater tendency of CC in high volumes (greater than $400 \mathrm{cc}$ ) and in smooth prostheses. The growth of periprosthetic tissue is considered to be greater in smooth prostheses than in textured prostheses, which causes multidirectional contractile forces with a tendency to neutralize each other. With round prostheses, a collapse of the upper pole can occur, which could favor the creation of pores and ruptures [10].

\section{Conclusions}

Prevention of post-augmentation CC after implantation is performed with the appropriate selection of patients, surgical technique, type of implant, and postoperative indications.

\section{Recommendations}

Properly select patients for augmentation Mastoplasty surgery according to national Plastic Surgery protocols. Identify avoidable or modifiable risk factors to avoid CC. Carry out future investigations of the immune system associated with CC to identify high-risk patients, whose prevention strategies would be personalized.

Avoid medical intrusion to avoid CC with its fatal economic and social consequences.

\section{References}

1. Shangshan L, Chen L, Wenyue L, Dali M, Luan (2018) Capsular contracture rate after breast augmentation with periareolar versus other two (inframammary and transaxillar) incisions: A meta-analysis. Aesth Plast Surg 42(1): 32-37.

2. Liu X, Zhou L, Gao Y, Yuan X, Fan D (2015) Comparison of the Postoperative Incidence Rate of Capsular Contracture among Diferent Breast Implants: A Cumulative MetaAnalysis. Plos One 10(2): e0116071.

3. Bachour Y, Verweij SP, Gibbs S, Johannes C, Ritt M, et al. (2018) The Aetiopathogenesis of capsular contracture: A systematic review of the literature. J Plast Reconstr Aesthet Surg 71(3): 307-317.

4. Pina Martinez V (2016) Etiological factors involved in the appearance of breast capsular contracture in patients undergoing cosmetic augmentation mammoplasty: a prospective observational study. Cir Plast Iberolatinoam 42(1): 13-20.

5. Headon H, Kasem A, Mokbel K (2015) Capsular contracture after breast augmentation: An update for clinical practice. Arch Plast Surg 42(5): 532-543.

6. Jeong T, Han J, Min K (2018) Treatment of capsular contracture after breast augmentation with serial fat grafting and implantation. Arch Aesthetic Plast Surg 24(2): 68-71.

7. Angulo Urteaga R (2019) Factors associated with the formation of capsular contracture in breast implant surgery clinic los andes 2007-2017 degree work. Academic repository USMP.

8. Vazquez G, Audoin F, Pellon A (2011) Microtrauma as an etiology of late seroma in augmentation mammoplasty. Cir Plast Iberolatinoam 37(3): 215-219.

9. Duteille F, Perrot P, Bacheley M, Stewart S (2017) Eightyear safety data for round and anatomical silicone gel breast implants. Aesthetic Surg J 38(2): 151-161.

10. Hendricks H (2007) Complete submuscular breast augmentation: 650 cases managed using an alternative surgical technique. Aesthetic Plast Surg 31(2): 147-153.

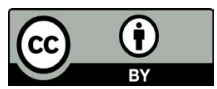

